\title{
金属有机骨架材料在放射性核素去除中的研究
}

\author{
王祥学，于淑君，王祥科 \\ (华北电力大学 环境科学与工程学院, 北京 102206)
}

摘 要: 核能利用的过程中, 从铀矿开采、核燃料加工、核能发电到乏燃料后处理, 会产生大量放射性废物, 部分放 射性核素会不可避免的释放到环境中，对环境和人类健康造成重大危害。放射性核素的高效去除是核电健康发展的 重要关键科学问题之一。近年来，高化学稳定性、具有大量功能基团而且结构可调的多孔金属有机骨架材料(MOFs) 在放射性污染治理方面受到国内外同行的高度关注。本文系统地介绍了 MOFs 及 MOFs 复合材料在放射性核素吸附 去除方面的研究进展，通过宏观吸附、模型分析、先进光谱表征和理论计算四个方面描述放射性核素与 MOFs 材料的 界面作用机理，并对 MOFs 材料的吸附性能与其它材料进行对比，评价 MOFs 材料在放射性污染治理中的应用前景。 关＼cjkstart键＼cjkstart词：金属有机骨架材料；放射性核素；吸附；分析表征；作用机理；综述 中图分类号: TQ174 文献标识码: A

\section{Removal of Radionuclides by Metal-organic Framework-based Materials}

WANG Xiang-Xue, YU Shu-Jun, WANG Xiang-Ke

(College of Environmental Science and Engineering, North China Electric Power University, Beijing 102206, China)

\begin{abstract}
With the development of nuclear energy, the long-lived radionuclides are inevitably released into the natural environment during the mine process, fuel manufacture, nuclear power usable and spent fuel management, which are dangerous to human health and environmental pollution. Thereby the efficient elimination of radionuclides is an important parameter which affects the development of nuclear power. In recent years, the metal-organic frameworks (MOFs) have attracted worldwide attention in the adsorption of radionuclides from large volume of aqueous solutions, because of their high chemical stability, abundant functional groups and changeable porous structures. In this review, we mainly summarized the recent works of MOFs in the efficient removal of radionuclides, and to understand the interaction mechanism from batch adsorption experiments, model analysis, advanced spectroscopy analysis, and theoretical calculation. The adsorption capacities of MOFs with other materials were also summarized, and the future research opportunities and challenges are given in the perspective.
\end{abstract}

Key words: metal-organic frameworks (MOFs); radionuclides; adsorption; analytical characterization; interaction mechanism; review

核能的快速发展会不可避免地造成大量放射性 核素释放到环境中, 给环境和人类健康造成重大危 害 ${ }^{[1]}$ 。放射性核素进入环境的主要途径包括: (1)铀矿
开采浓缩过程中产生的废矿、废水处置不当, ${ }^{238} \mathrm{U}$ 及 其衰变子体 $\left({ }^{230} \mathrm{Th}\right.$ 和 $\left.{ }^{226} \mathrm{Ra}\right)$ 随降水迁移到附近的土 壤和水体中，造成环境污染; (2)乏燃料后处理以及

收稿日期：2018-05-07; 收到修改稿日期：2018-06-08

基金项目：国家自然科学基金(21577032); 国家重点基础研究发展计划(2017YFA0207002); 中央高校基本科研业务费专项 基金(2018MS114, 2018ZD11)

National Natural Science Foundation of China (21577032); National Key Research and Development Program of China (2017YFA0207002); Fundamental Research Funds for the Central Universities (2018MS114, 2018ZD11)

作者简介: 王祥学(1976-), 男, 讲师. E-mail: xxwang@ncepu.edu.cn

通讯作者: 王祥科, 教授. E-mail: xkwang@ncepu.edu.cn 
高放核废物存放过程中由于设计和管理缺陷导致的 放射性物质外泄，与之相关的典型核污染场如美国 的汉福德地区, 代表性污染核素除 ${ }^{238} \mathrm{U}$ 和 ${ }^{239} \mathrm{Pu}$ 外, 还有相当量的弱吸附性核素如 ${ }^{99} \mathrm{Tc}$ 以及长寿命、高 毒性的次钶系核素(如 ${ }^{237} \mathrm{~Np} 、{ }^{241} \mathrm{Am}$ 和 ${ }^{242} \mathrm{Cm}$ 等); (3) 核电站以及其它民用核设施在生产过程中发生安全 事故，造成的突发性放射性物质泄漏，例如 1986 年 4 月 26 日的切尔诺贝利核事故以及 2011 年 3 月 11 日的福岛核事故，释放的典型放射性核素如 ${ }^{137} \mathrm{Cs}$ 、 ${ }^{90} \mathrm{Sr}$ 和 ${ }^{131} \mathrm{I}$ 等; (4)核试验以及军用核武器的使用也会 造成大面积的放射性污染 ${ }^{[2-4]}$ 。长寿命放射性核素与 有机污染物和重金属离子相比，放射性核素不能降 解且在生物体内容易积累, 放射性核素的毒性无法 与一般污染物的化学毒性那样可通过一系列化学反 应将其转化消除, 特别是其放射性自发衰变所带来 的放射性辐照效应可使人体器官发生病变, 因此放 射性污染通过各种途径进入到人体内会对公众健康 产生严重危害造成内辐照伤害, 相对于重金属离子, 放射性核素由于其放射性衰变造成的危害比重金属 离子高 3 到 4 个数量级 ${ }^{[5-8]}$ 。

在过去的几十年中，各种方法包括吸附法、电 渗析法、化学沉淀法、蒸发回收、超滤法、萃取法、 光催化降解、氧化/还原、生物处理、膜技术等应用 于放射性废水中核素的高效去除，但是这些方法大 多有一定的缺点, 如沉淀法容易产生大量的污泥, 光催化降解会产生一些副产物, 吸附法由于操作简 单、成本低、可以大规模使用等优点在放射性污染 治理中得到了广泛应用 ${ }^{[9-12]}$ 。传统的吸附材料如粘 土矿物、氧化物、纳米零价铁、水滑石、活性炭、 纳米碳材料等已经被广泛应用于放射性核素的高效 去除, 但是这些材料普遍存在以下一个或者几个缺 点，如(1)吸附速度慢; (2)选择性差; (3)小孔径或者 孔隙体积小; (4)再生能力差或者不能重复使用; (5) 较差的热、化学或者辐射稳定性; (6)吸附能力低; (7)制 备复杂或者不能低成本大规模制备等 ${ }^{[13-15]}$ 。因此, 针对放射性废水中目标核素的特点, 通过设计和构筑 对目标核素具有高选择性的材料, 实现对复杂体系中 低浓度放射性核素的高效去除，具有重要的科学意义。

金属有机骨架材料(Metal-organic Frameworks, MOFs) 是一类通过配位键连接的有机配体组成的多 孔晶体材料, 由于其具有很高的化学稳定性、表面 具有大量功能基团而且结构可调, 受到了全球研究 者的广泛关注 ${ }^{[16]}$ 。作为吸附材料, 已经广泛应用于 各种金属离子如 $\mathrm{Fe}(\mathrm{III}) 、 \mathrm{Cu}(\mathrm{II}) 、 \mathrm{Ca}(\mathrm{II}) 、 \mathrm{Al}(\mathrm{III})$ 、 $\mathrm{Mg}(\mathrm{II}) 、 \mathrm{Zn}(\mathrm{II}) 、 \mathrm{Cd}(\mathrm{II}) 、 \mathrm{Co}(\mathrm{II}) 、 \mathrm{Zr}(\mathrm{IV}) 、 \mathrm{Ln}(\mathrm{III})$ 和 放射性核素如 Tc(VII)、Eu(III)、U(VI)、Th(IV)等高
选择性去除 ${ }^{[17]}$ 。MOFs 材料的吸附性能, 如吸附动 力学、吸附能力、吸附热力学、选择性、化学稳定 性、辐照稳定性、循环利用等, 受 MOFs 材料的骨 架结构、表面亲水性、表面积、表面功能基团和孔 径大小等影响明显 ${ }^{[18]}$ 。为了提高 MOFs 材料的吸附 能力和吸附选择性，通常采用以下几种办法提高 MOFs 材料的性能，如(1)利用大的有机配体扩大 MOFs 的孔径, 促进放射性核素在 MOFs 材料中的 扩散; (2)设计有缺陷的 MOFs, 通过缺陷作用提高吸 附位点数量; (3)增加有吸附络合能力的有机配体, 如增加 $-\mathrm{NH}_{2} 、-\mathrm{OH}$ 和- $\mathrm{SH}$ 等与放射性核素具有 Lewis 酸碱反应络合能力强的功能基团，提高对放射性核 素的吸附和选择性; (4)MOFs 与其它材料的有效复合, 如与磁性材料的有效复合, 可以利用磁性从溶液中 分离回收 MOFs 材料等 ${ }^{[19-21]}$ 。

本文从宏观吸附、模型分析、先进光谱技术表 征和理论计算四个角度, 总结近几年 MOFs 材料在 环境放射性污染治理方面的应用研究进展, 并对其 未来前景进行展望。

\section{1 宏观吸附实验}

静态吸附实验是目前应用最广泛的技术，该方 法通过分析放射性核素在 MOFs 材料上的吸附规律, 可以直接得到最大吸附容量和吸附动力学平衡等关 键数据 ${ }^{[22-24]}$ 。

MOFs 作为吸附材料最早应用于放射性核素吸 附的例子是 UiO-68 型材料(MOF-2 和 MOF-3), 在 $\mathrm{pH}=2.5$ 的水溶液和模拟海水中 MOF-2 对 U(VI) 的吸 附量达到了 217 和 $188 \mathrm{mg} / \mathrm{g}$, DFT 计算证明了 U(VI) 离子与磷酰脲功能基团形成了强络合物, U(VI)离子 与两个磷酰氧基团形成配位增加了 UiO-68 对铀酰 离子在酸性条件下的吸附能力 ${ }^{[25]}$ 。中国科学院高能 物理研究所石伟群等 ${ }^{[26]}$ 制备了三种氨基功能化的 MIL-101 材料包括 MIL-101-NH $2 、$ MIL-101-ED(ED：乙 二胺)和 MIL-101-DETA(DETA：二亚乙基三胺), 表 征研究(图 1)发现制备的材料具有八面体形貌、良好 的结晶性、丰富的官能团和较大的表面积。静态批实 验结果表明 U(VI)的最大吸附能力为 MIL-101-DETA $(350 \mathrm{mg} / \mathrm{g})>$ MIL-101-ED $(200 \mathrm{mg} / \mathrm{g})>\mathrm{MIL}-101-\mathrm{NH}_{2}$ $(90 \mathrm{mg} / \mathrm{g})>\mathrm{MIL}-101(20 \mathrm{mg} / \mathrm{g})$, 表明氨基能够有效提 高 MOFs 材料对放射性核素的去除效率和吸附能 力。铀酰离子在溶液中可以看作是一种强路易斯酸, 对氢氧根和羧酸分子具有很强的亲和能力, 因此羧 基化的 MOFs 材料能够有效提高 MOFs 材料对铀酰 离子的吸附能力, 饱和吸附量达到了 $314 \mathrm{mg} / \mathrm{g}$, 并 且其它共存金属离子对铀酰离子的吸附干扰非常弱 ${ }^{[27]}$ 。 
动力学实验可以提供吸附过程的作用情况, 苏州大 学王设凹等 ${ }^{[28]}$ 利用 SCU-101 去除 $\mathrm{TcO}_{4}^{-}$, 结果表明 在 $5 \mathrm{~min}$ 内吸附量达到 $85 \%, 10 \mathrm{~min}$ 后超过 $95 \%$ 的 $\mathrm{TcO}_{4}{ }^{-}$被吸附(图 2(b)), 速度远高于商业离子交换树 脂(A532E 和 A530E), 主要是由于 $\mathrm{TcO}_{4}{ }^{-}$在 $\mathrm{SCU}-101$
孔道内的快速扩散, 对 $\mathrm{ReO}_{4}^{-}$的最大吸附能力达到 $217 \mathrm{mg} / \mathrm{g}$ (图 2(c))。MOFs 材料 SCU-100 和 SCU-101 中孔道内的 $\mathrm{NO}_{3}^{-}$可以交换去除 $\mathrm{TcO}_{4}^{-}$和 $\mathrm{ReO}_{4}^{-}$, 该 材料具有高选择性(图 2(d) (e))和良好的耐辐照稳 定性(图 2(f) $)^{[28-29]}$ 。
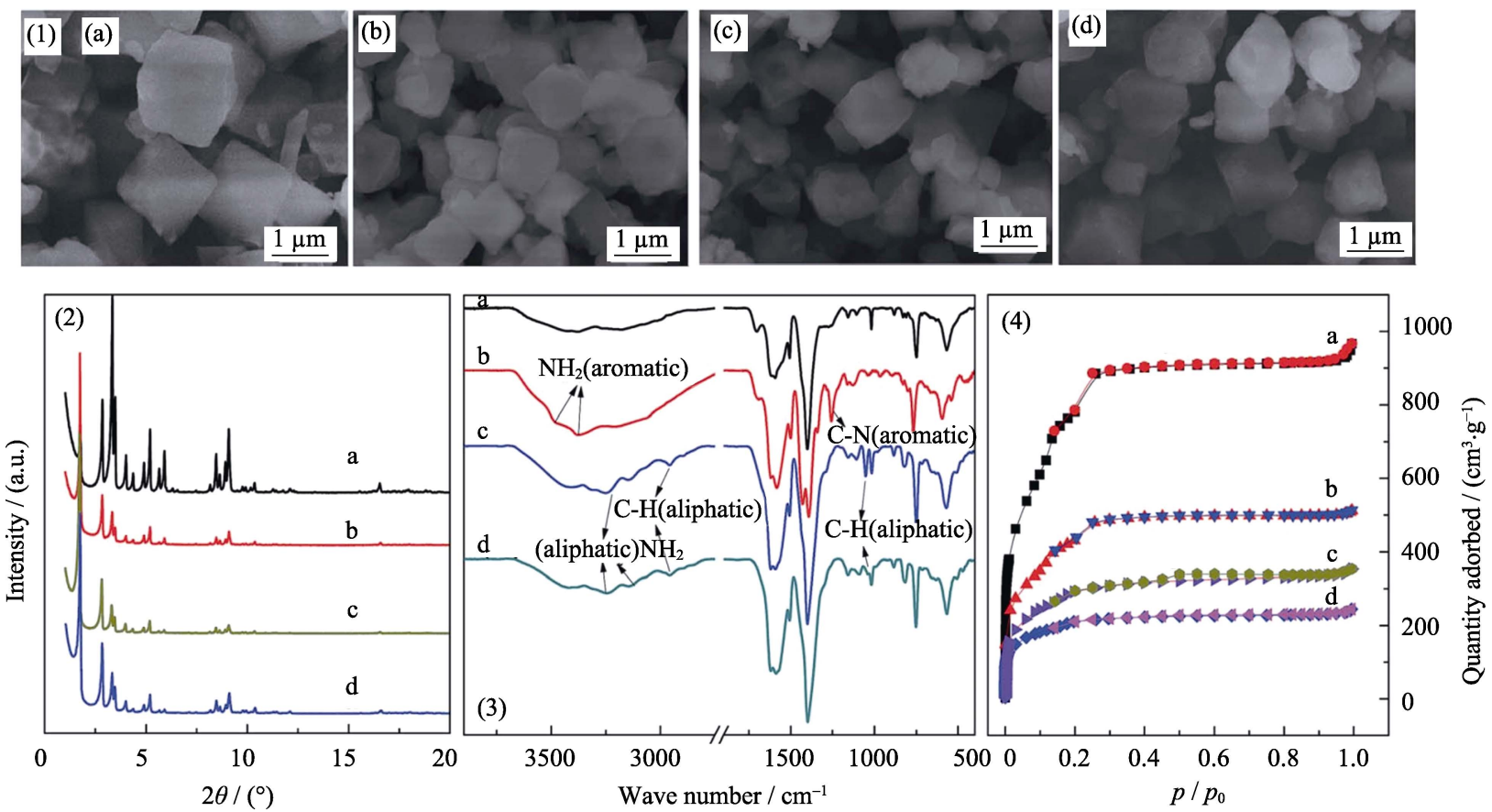

图 1 MIL-101 及其氨基衍生物的 SEM 照片(1), XRD 图谱(2), FT-IR 谱图(3)和 $\mathrm{N}_{2}$ 吸脱附等温线(4) ${ }^{[26]}$

Fig. 1 (1) SEM images, (2) XRD patterns, (3) FT-IR spectra, (4) $\mathrm{N}_{2}$ sorption isotherms ${ }^{[26]}$ of MIL-101 and its amino derivatives, (a) MIL-101; (b) MIL-101-NH $\mathrm{NH}_{2}$; (c) MIL-101-ED; (d) MIL-101-DETA
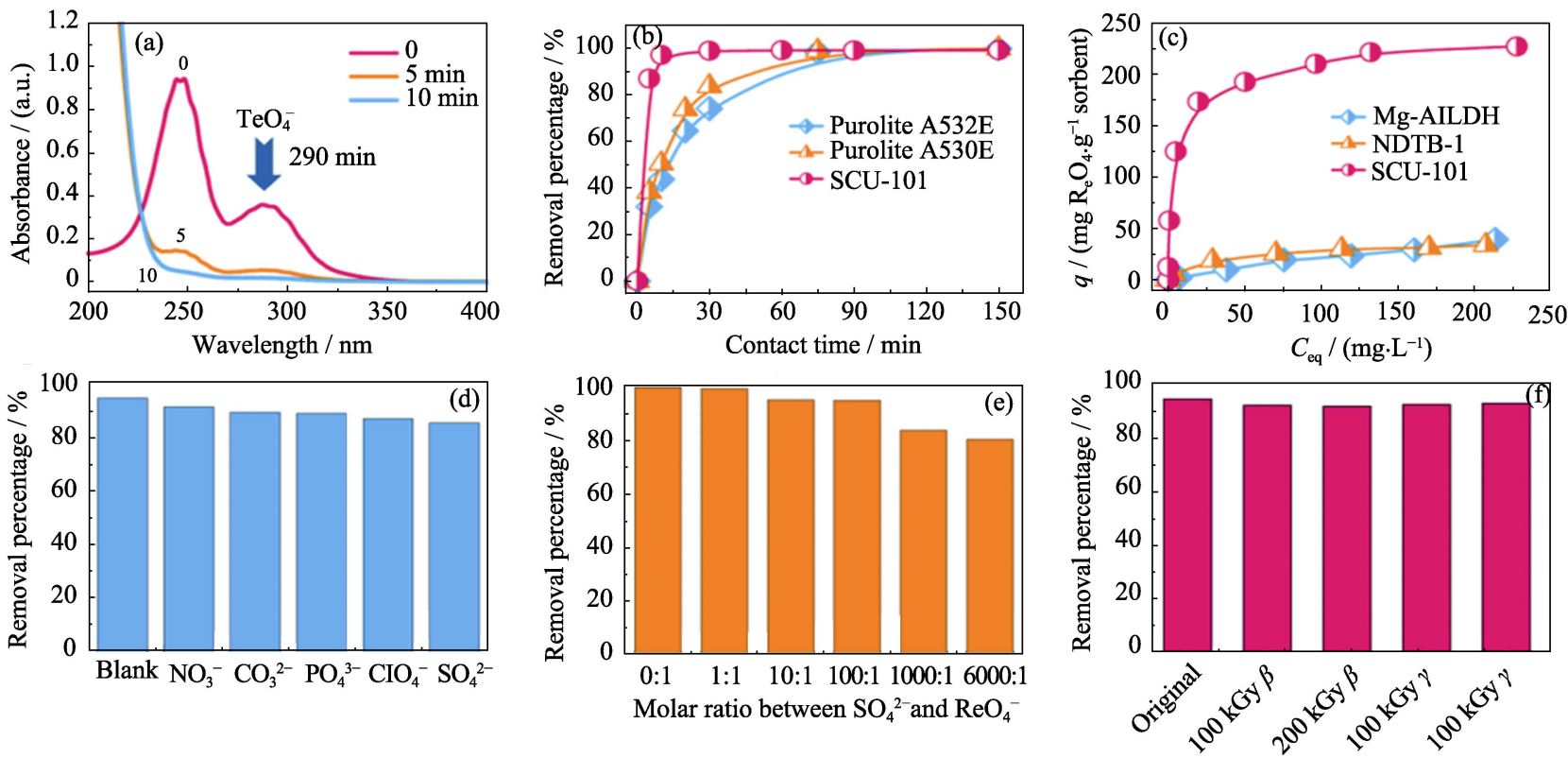

图 2 (a) $\mathrm{TcO}_{4}{ }^{-}$的 UV-Vis 吸收光谱; (b) $\mathrm{TcO}_{4}{ }^{-}$在 SCU-101、Purolite $\mathrm{A} 530 \mathrm{E}$ 和 $\mathrm{A} 532 \mathrm{E}$ 上的吸附动力学;

(c) $\mathrm{ReO}_{4}{ }^{-}$在 SCU-101、Mg-Al-LDH 和 NDTB-1 上的吸附等温线; (d) 阴离子对 $\mathrm{TcO}_{4}{ }^{-}$去除效果的影响; (e) $\mathrm{SO}_{4}{ }^{2-}$ 对 $\mathrm{ReO}_{4}{ }^{-}$和 $\mathrm{SCU}-101$ 离子交换的影响; (f)SCU-101 样品经过多次辐照之后对 $\mathrm{ReO}_{4}{ }^{-}$的去除效果 ${ }^{[28]}$

Fig. 2 (a) UV-Vis absorption spectra of $\mathrm{TcO}_{4}^{-}$during the anion exchange; (b) Sorption kinetics of $\mathrm{TcO}_{4}^{-}$by SCU-101 compared with Purolite A530E and A532E; (c) Sorption isotherms of $\mathrm{ReO}_{4}^{-}$by SCU-101, Mg-Al-LDH, and NDTB-1;

(d) Effect of competing anions on the removal percentage of $\mathrm{TcO}_{4}{ }^{-}$by SCU-101; (e) Effect of $\mathrm{SO}_{4}{ }^{2-}$ on the anion exchange of $\mathrm{ReO}_{4}{ }^{-}$by SCU-101; (f) Removal percentage of $\mathrm{ReO}_{4}^{-}$after irradiation as compared with the original SCU-101 sample ${ }^{[28]}$ 
表 1 总结了不同的放射性核素在 MOFs 材料和 其它常用材料上的吸附性质。不同的 MOFs 材料对 放射性核素表现出了不同的吸附能力, 这与周围环 境条件、吸附剂本身的结构性质以及吸附质性质相 关。此外, 与其它常用吸附剂如粘土矿物、石墨烯 材料和金属氧化物材料相比, MOFs 材料展现出更 高的吸附量、选择性和去除效率, 这主要归因于其 较高的孔隙率、较大的表面积、孔径可调以及拓扑 结构多样性等优势。广泛研究表明 MOFs 材料在放 射性废水处理方面展现了优异的性能, 能够作为核 废料的潜在处理材料。

\section{2 模型计算}

为了对吸附动力学数据进行准确的分析模拟, 大量的动力学模型用来模拟吸附动力学数据如假一 阶模型 (Pseudo-first-order model), 假二阶模型 (Pseudo-second-order model), 扩散模型(Intraparticle diffusion model), Lagergren 模型, Zeldowitsch 模型和 Elovich 模型等 ${ }^{[41-43]}$ 。Naeimi 等 ${ }^{[44]}$ 利用假一阶模型 (图 3(a))、假二阶模型(图 3(b))、扩散模型(图 3(c))
和 Elovich 模型(图 3(d)) 模拟计算了 $\mathrm{Cs}^{+}$吸附到 $\mathrm{MOF} / \mathrm{KNiFC}$ 和 $\mathrm{MOF} / \mathrm{Fe}_{3} \mathrm{O}_{4} / \mathrm{KNiFC}$ 上的动力学数据, 结果表明假二阶模型对数据拟合最好, 表明吸附主 要受材料表面的化学作用位点控制, 吸附过程是化 学吸附为主。

对吸附等温线的模型分析, 可以评价吸附机理, Langmuir, Freundlich, Brunauer-Emmett-Teller, RedlichPeterson, Dubinin-Radushkevich (D-R), Temkin, Toth, Koble-Corrigan, Sips, Flory-Huggins 和 RadkePrausnitz 等吸附等温线模型可以从不同角度分析放 射性核素与 MOFs 材料的界面作用机理, 如 Langmuir 模型表明吸附是单层吸附 ${ }^{[45-47]}$ 。如图 3(e) (h), U(VI) 在 UiO-66 和 GO-COOH/UiO-66 上的吸附模型模拟 结果表明 $U(V I)$ 的吸附主要是单层吸附, D-R 模型表 明吸附主要是化学吸附为主 ${ }^{[30]}$ 。

除吸附量和吸附机理外, 吸附热力学参数如吉 布斯自由能变化, 焓变和熵变等热力学数据可以从 不同温度条件下的吸附等温线拟合得到, 这些参数 对了解放射性核素与 MOFs 材料的相互作用, 吸附 过程是否自发以及吸附过程是吸热/放热等具有重 要的参考意义, 很多文献有相关热力学参数分析报道。

表 1 放射性核素在不同材料上的去除效果和作用机理

Table 1 Radionuclides adsorption on different materials

\begin{tabular}{|c|c|c|c|c|c|c|c|c|}
\hline Adsorbents & Radionuclides & $\mathrm{s}(\mathrm{m} / \mathrm{V}) /\left(\mathrm{g} \cdot \mathrm{L}^{-1}\right)$ & $C_{0} /\left(\mathrm{mg} \cdot \mathrm{L}^{-1}\right)$ & $t / \mathrm{h}$ & $\mathrm{pH}$ & $Q_{\max } /\left(\mathrm{mg} \cdot \mathrm{g}^{-1}\right)$ & Interaction mechanism & Ref. \\
\hline MIL-101 & $\mathrm{U}(\mathrm{VI})$ & 0.4 & 100 & 2 & 5.5 & 20 & Surface complexation & {$[26]$} \\
\hline MIL-101-NH 2 & $\mathrm{U}(\mathrm{VI})$ & 0.4 & 100 & 2 & 5.5 & 90 & Surface complexation & {$[26]$} \\
\hline MIL-101-ED & $\mathrm{U}(\mathrm{VI})$ & 0.4 & 100 & 2 & 5.5 & 200 & Surface complexation & {$[26]$} \\
\hline MIL-101-DETA & $\mathrm{U}(\mathrm{VI})$ & 0.4 & 100 & 2 & 5.5 & 350 & Surface complexation & {$[26]$} \\
\hline GO-COOH/UiO-66 & $\mathrm{U}(\mathrm{VI})$ & 0.5 & 95 & 4 & 8.0 & 188 & Surface complexation and ion exchange & {$[30]$} \\
\hline SCU-101 & $\operatorname{Re}(I V)$ & 1.0 & 1000 & 0.2 & - & 217 & Ion exchange & {$[28]$} \\
\hline SCU-100 & $\operatorname{Re}(I V)$ & 1.0 & 28 & 2 & - & 541 & Ion exchange & [29] \\
\hline $\mathrm{UiO}-66-(\mathrm{COOH})_{2}$ & $\operatorname{Th}(\mathrm{IV})$ & 0.4 & 100 & 6 & 3.0 & 350 & Surface complexation & {$[31]$} \\
\hline $\mathrm{MOF}-808-\mathrm{SO}_{4}$ & $\mathrm{Ba}(\mathrm{II})$ & 1.0 & 42 & 0.1 & 5.8 & 131 & Surface complexation & {$[32]$} \\
\hline UiO-66-Schiff & $\mathrm{Co}(\mathrm{II})$ & 0.1 & 10 & 5 & 8.4 & 256 & Surface complexation & [33] \\
\hline FJSM-InMOF & $\operatorname{Sr}(\mathrm{II})$ & 2.5 & 18 & 12 & - & 44 & Ion exchange & [34] \\
\hline FJSM-InMOF & $\mathrm{Cs}(\mathrm{I})$ & 2.5 & 90 & 3 & - & 199 & Ion exchange & [34] \\
\hline LDO-C & $\mathrm{U}(\mathrm{VI})$ & 0.1 & 50 & 4 & 5.0 & 354 & Surface complexation and ion exchange & {$[35]$} \\
\hline CS@LDH & $\mathrm{U}(\mathrm{VI})$ & 0.2 & 41 & 3 & 5.0 & 157 & Surface complexation & {$[36]$} \\
\hline GO & $\mathrm{Co}(\mathrm{II})$ & 0.1 & 10 & 4 & 5.0 & 44 & Surface complexation & {$[37]$} \\
\hline $\mathrm{LDH}$ & $\mathrm{U}(\mathrm{VI})$ & 0.2 & 50 & 6 & 4.5 & 69 & $\begin{array}{c}\text { Surface complexation and electrostatic } \\
\text { interaction }\end{array}$ & {$[38]$} \\
\hline Na-montmorillonite & $\mathrm{Ni}(\mathrm{II})$ & 0.5 & 10 & 6 & 6.0 & 13 & Surface complexation and ion exchange & {$[39]$} \\
\hline $\mathrm{Fe}_{3} \mathrm{O}_{4} @ \mathrm{TNS}$ & $\mathrm{U}(\mathrm{VI})$ & 0.2 & 20 & 8 & 5.0 & 83 & Ion exchange & {$[40]$} \\
\hline
\end{tabular}



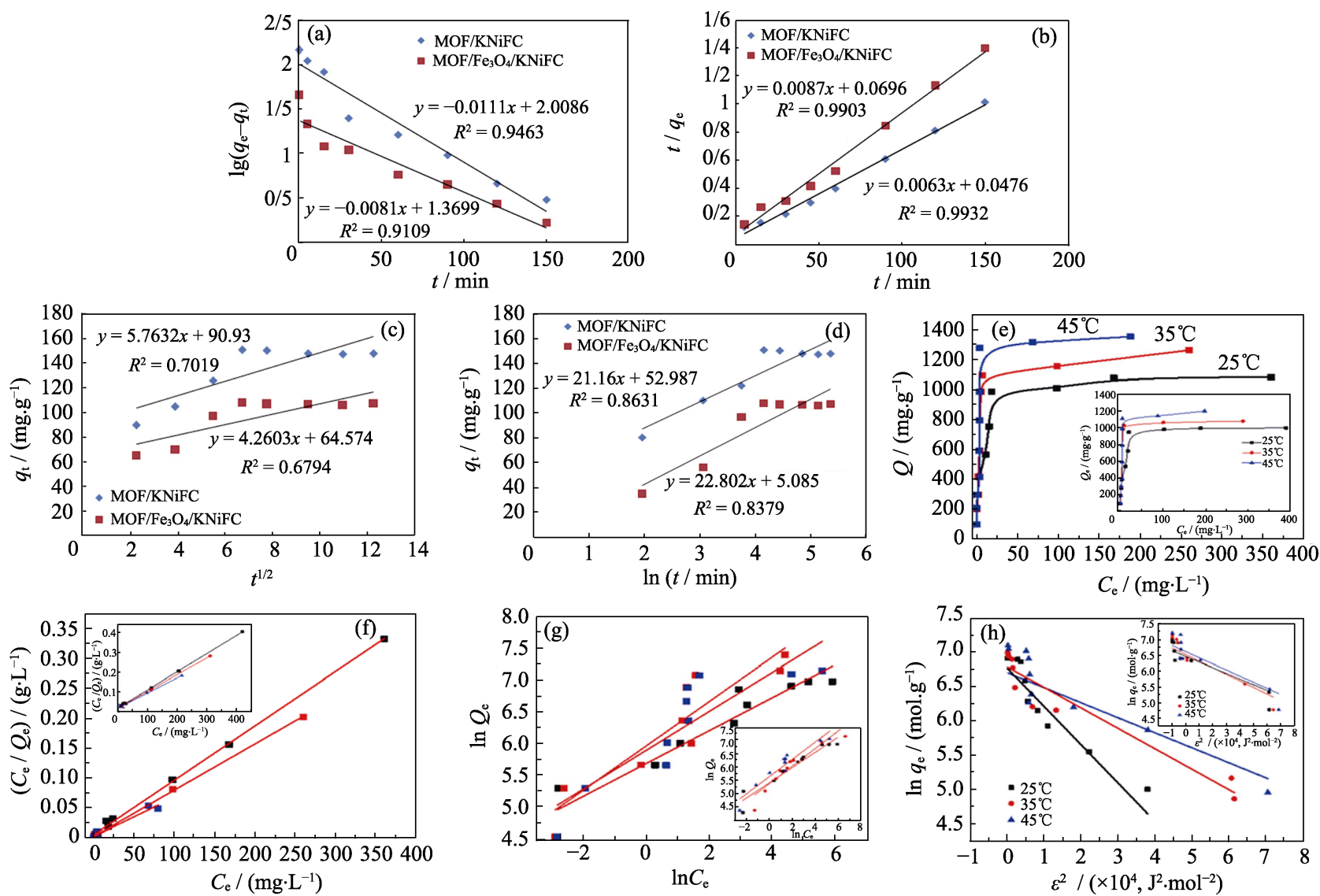

图 $3 \mathrm{Cs}^{+}$吸附到 $\mathrm{MOF} / \mathrm{KNiFC}$ 和 $\mathrm{MOF} / \mathrm{Fe}_{3} \mathrm{O}_{4} / \mathrm{KNiFC}$ 上的假一阶模型(a)、假二阶模型(b)、扩散模型(c)和 Elovich 模型(d)拟合 ${ }^{[44]}$; (e)U(VI)在 UiO-66(插图)和 GO-COOH/UiO-66 复合物上的吸附等温线:

(f)Langmuir 模型, (g)Freundlich 模型, (h)Dubinin-Radushkevich 模型 ${ }^{[30]}$

Fig. 3 Linear pseudo-first-order kinetic (a), pseudo-second-order (b), intraparticle diffusion (c) and elovich equation (d) for adsorption of $\mathrm{Cs}^{+}$on $\mathrm{MOF} / \mathrm{KNiFC}$ and $\mathrm{MOF} / \mathrm{Fe}_{3} \mathrm{O}_{4} / \mathrm{KNiFC}^{[44]}$; (e) Isotherm model of $\mathrm{U}(\mathrm{VI})$ adsorption on UiO-66 (inset) and

GO-COOH/UiO-66 composites; (f) Langmuir model, (g) Freundlich model, and (h) Dubinin-Radushkevich model ${ }^{[30]}$

\section{3 先进光谱技术表征}

先进光谱技术如 X 射线吸收精细结构光谱 (X-ray Absorption Fine Structure (XAFS) Spectroscopy)、X 射线光电子能谱 (X-ray Photoelectron Spectroscopy (XPS)) 和傅里叶红外光谱 (Fourier Transformed Infrared (FT-IR) Spectroscopy)等可以在 分子水平上给出放射性核素与功能基团的界面作 用、配位数、键长等形态和微观结构信息, 给出与 不同功能基团的相互作用, 这些信息对了解和分析 放射性核素与 MOFs 材料的界面作用、表面(孔径) 结合性质具有重要意义 ${ }^{[48-51]}$ 。

XPS 技术可以对表面和近表面的放射性核素提 供氧化/还原态、配位模式、功能基团络合等方面的 信息，在样品表面，可以检测到 3 30 个原子层深度 的放射性核素，对表征放射性核素和 MOFs 之间的 吸附反应可以定性、半定量给出表征数据 ${ }^{[52-53]}$ 。XPS 的缺点是测量需要在真空条件中, 可能会影响样品 的性能。XAFS 包括 X 射线吸收近边结构光谱(X-ray
Absorption Near-edge Structure (XANES) Spectroscopy) 和扩展 $\mathrm{X}$ 射线吸收精细结构光谱(Extended X-ray Absorption Fine Structure (EXAFS) Spectroscopy), 在 分子水平上给出配位数、键长等微观结构信息。如 图 4, U(VI)在 MIL-101 的吸附样品中的 XANES 光 谱测量结果表明铀酰存在双雉体骨架结构, U(VI)主 要与 MIL-101 材料中的胺基配位 ${ }^{[54]}$ 。XAFS 对放射 性核素的微观结构分析的优点是非常准确直观地给 出放射性核素的配位和键长情况，从而可以判断放 射性核素在材料表面上的存在形态和微观结构, 准 确分析放射性核素与材料的界面作用机理。缺点是 测量需要同步辐射光源，用光时间非常紧张，而且 样品分析需要很专业的人员才能给出非常准确的分 析结果。

每一种光谱技术都有独特的优势，但没有一个 光谱技术是万能的，通常几种光谱技术的结合可以 更加准确的对吸附形态和吸附机理给出判断, 如 FT-IR 和 XPS 结合可以确定吸附发生的作用位点和 功能基团配位情况; FT-IR 和 EXAFS 技术结合对 

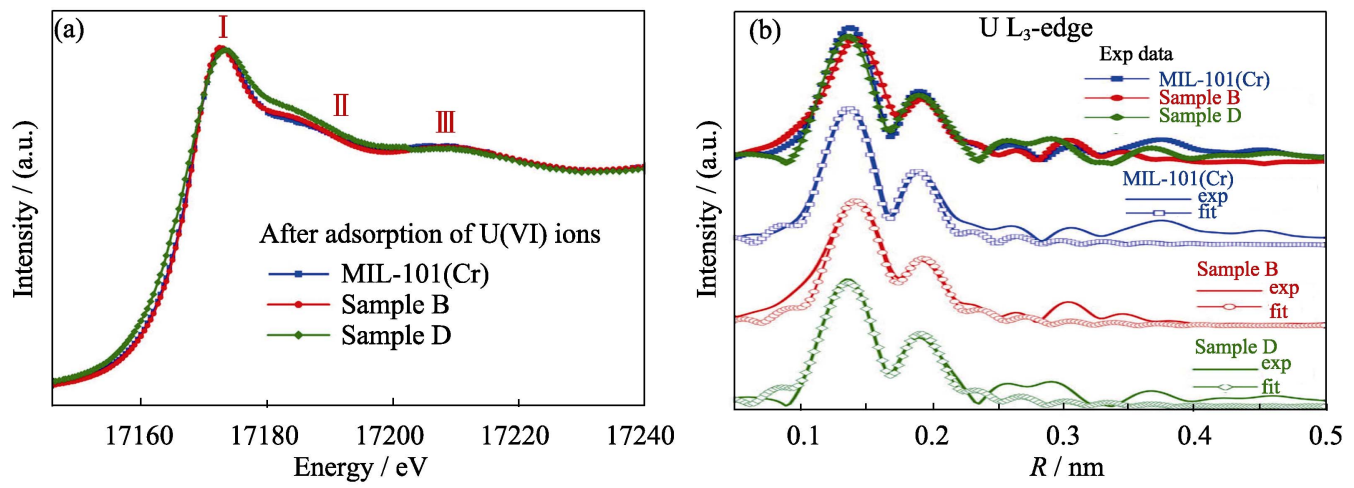

图 4 (a)原始的 MIL-101(Cr)和不同乙二胺(ED)改性 ED-MIL-101(Cr)材料吸附 U(VI)之后的 $\mathrm{U} \mathrm{L}_{3}$-edge XANES 光谱, (b)不同样品对应的傅里叶转换光谱图 ${ }^{[54]}$

Fig. 4 (a) Comparison of experimental $\mathrm{U} \mathrm{L}_{3}$-edge XANES spectra for pristine MIL-101(Cr), and different ED contents grafting ED-MIL-101(Cr) samples after the adsorption of U(VI), (b) Experimental Fourier transform of the $\mathrm{U} \mathrm{L}_{3}$-edge EXAFS data for different samples and their corresponding fits ${ }^{[54]}$

U(VI)在 MIL-101-NH $、$ MIL-101-ED 和 MIL-101DETA 的分析表明, 在 U(VI)的吸附过程中并没有伴

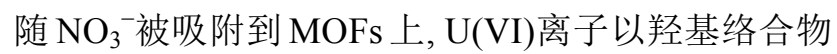
的形式吸附在 MOFs 上, EXAFS 的测量结果表明 $\mathrm{U}-\mathrm{Oax}$ 键长为 $0.179 \mathrm{~nm}, \mathrm{U}$ 离子第一壳层有 4 个 $\mathrm{O}$ 原子和 1 个 $\mathrm{N}$ 原子 ${ }^{[26]}$ 。除了上述技术，其它技术如 单晶 $\mathrm{X}$ 射线衍射、透射电镜、拉曼光谱等技术也可以 表征放射性核素在 MOFs 材料表面的吸附行为 ${ }^{[52-55]}$ 。

\section{4 理论计算方法}

为了深层次理解放射性核素与 MOFs 材料相互 作用, 理论计算如密度泛函理论(Density Functional Theory, DFT)和分子模拟(Molecular Simulation)可以 提供结构、反应能量变化、电荷密度等实验和光谱 表征无法给出的数据, 帮助深层次了解作用机理, 指导 MOFs 材料的设计和构筑, 优化放射性核素的 吸附, 具有重要的参考意义 ${ }^{[1,56-57]}$ 。DFT 计算是对 实验和光谱表征数据的有力解释和补充, 如 DFT 计 算表明 $\mathrm{U}(\mathrm{VI})$ 与质子化配体的结合比去质子化配体 的结合更稳定, 可以有力的解释酸性条件下 MOF-2 和 MOF-3 对 U(VI)离子在酸性条件下的强吸附能 力 $^{[25]}$ 。DFT 还可以提供稳定的构型、总吸附能等信 息, Co(II)与 UiO-66-Schiff 的席夫碱配体之间的络 合模型计算表明, Co(II)在 MOFs 材料表面形成了 $\left[\mathrm{Co}\left(\mathrm{H}_{2} \mathrm{O}\right)_{6}\right]^{2+}$ 的水合物, $\mathrm{Co}-\mathrm{O}$ 键的键长为 0.2169 和 $0.2162 \mathrm{~nm}$, 揭示了 $\mathrm{Co}(\mathrm{II})$ 的吸附是 $\left[\mathrm{Co}\left(\mathrm{H}_{2} \mathrm{O}\right)_{6}\right]^{2+}$ 和配 体的交换过程 ${ }^{[33]}$ 。

分子模拟可以提供吸附过程中吸附的位置和势 能等实验无法给出的数据, 在过去的十年中, 分子 模拟已经广泛应用于 MOFs 材料对气体分子的吸附
模拟计算中，对金属离子的吸附有一些模拟，但是 对放射性核素的吸附工作非常少。U(VI)与 SZ-2 之 间的分子动力学模拟结果 (图 5) 铀酰离子周围的赤 道水分子发挥着重要作用, 通过和 SZ-2 主框架上的 氢键受体如 $\mathrm{F}$ 和 $\mathrm{O}$ 原子形成氢键和范德华力从而将 $\mathrm{U}(\mathrm{VI})$ 稳定的结合在 SZ-2 材料表面和内部孔道 ${ }^{[58]}$ 。 与气体吸附模拟计算相比, MOFs 材料对放射性核 素吸附动力学模拟计算研究较少, 随着计算能力的 增强, 预计在这一领域会有很多的分子模拟计算。 表 2 总结了每个方法的优缺点、应用范围等情况。

\section{5 结束语}

MOFs 材料的制备和构筑已经有很多相关综述, 本文对 MOFs 材料在放射性核素的去除性质及作用 机理方面的最新研究进展, 从宏观吸附、模型分析 到先进光谱表征和理论计算做了比较全面的回顾。

MOFs 材料独特的物理化学性质, 超高的孔隙率和 稳定的骨架结构, 在放射性污染治理方面显示出独 特的优势, 如吸附速率快、吸附能力强、良好的选 择性和可重复利用等, 决定了 MOFs 材料在未来放 射性污染治理中广泛的应用前景。MOFs 材料对放 射性核素的吸附主要归结于表面功能基团与放射性 核素的络合作用、孔道的有效调控与放射性核素离 子半径的有效匹配等作用。尽管 MOFs 材料在放射 性污染治理方面已经开展了大量工作, 但是仍然面 临诸多挑战，如:

(1) 从宏观吸附角度考虑, 溶液的条件如 $\mathrm{pH}$ 值、 离子强度、共存干扰离子、天然有机物等对 MOFs 材料表面性质的影响和对放射性核素吸附的影响, 虽然有很多工作, 但是比较系统的研究还是不够全面。 
(a)

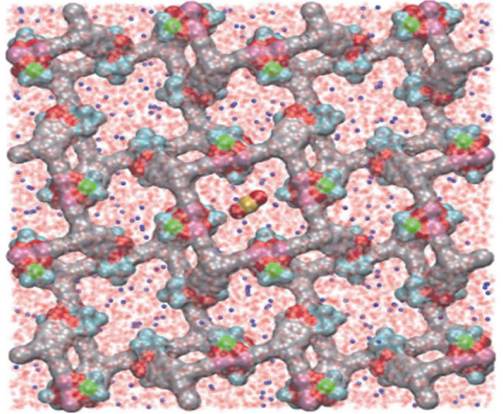

(b)

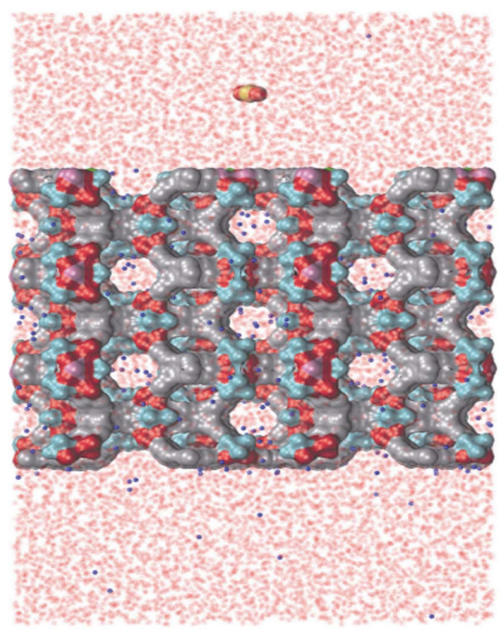

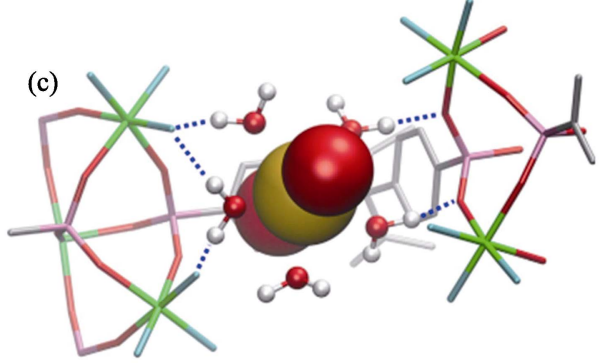
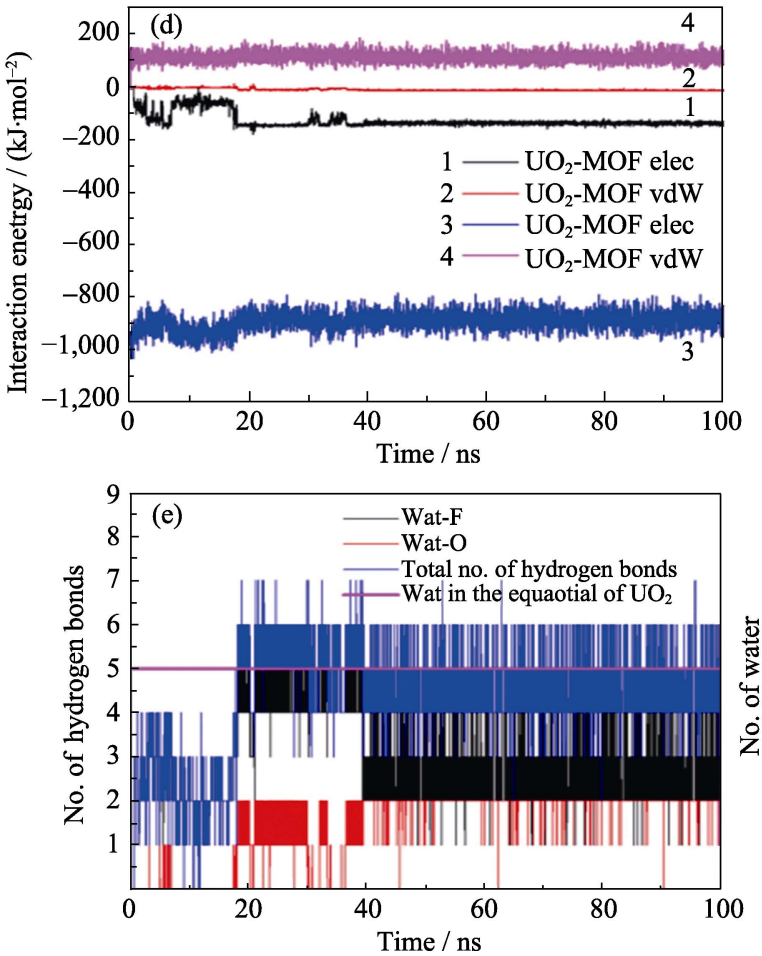

图 $5 \mathrm{U}(\mathrm{VI})$ 在 SZ-2 吸附的分子动力学模拟。 $\mathrm{U}(\mathrm{VI})$ 接近 SZ-2 [001]晶面 $c$ 轴的(a)俯视和(b)侧视图;

(c) $\mathrm{U}(\mathrm{VI})$ 结合到 $\mathrm{SZ}-2$ 上运行完一次(总共六次)的快照图(蓝色虚线代表赤道水分子和悬挂的氢键受体之间的氢键);

(d)时间对 U(VI)与 SZ-2 和水分子之间的静电作用和范德华作用的影响; (e)时间对铀酰离子赤道水分子的数量

(粉红色曲线)和赤道水分子与其它受体(包括主框架中的 $\mathrm{F}$ 和 $\mathrm{O}$ )之间形成的氢键数量的影响 ${ }^{[58]}$

Fig. 5 MD simulations on the process of uranyl sorption into SZ-2. The top (a) and side (b) view of the simulation system-1 (uranyl cation approaching along the $c$ axis); (c) The final snapshot (at $t \frac{1 / 4}{100} \mathrm{~ns}$ ) of run 1 (out of total 6) to show the importance of equatorial water of uranyl cation in mediating its binding to the SZ-2 (the blue dash line indication the hydrogen bond between equatorial water molecules and the dangling hydrogen bond acceptors); (d) Time evolution of the electrostatic and vdW interaction energies of uranyl cation with SZ-2 and water; (e) The number of equatorial water molecules of uranyl cation (pink curve) and the number of hydrogen bonds formed between equatorial coordinating water molecules and other acceptors (including $\mathrm{F}$ and $\mathrm{O}$ in main framework) as the function of simulation time ${ }^{[58]}$

（2）部分 MOFs 材料的活性 $\mathrm{pH}$ 范围比较窄，考 虑到放射性废水的特点, 设计构筑活性范围广的 MOFs 材料是未来研究的一个挑战和方向。

（3）虽然部分 MOFs 材料吸附速率快, 但是与其 它纳米材料相比, 部分 MOFs 材料吸附速率还是比 较慢, 主要与 MOFs 材料的孔径有关, 在未来实际废 水处理中, MOFs 材料的快速吸附对实际废水的处理 至关重要。

(4) MOFs 材料吸附大量放射性核素后，放射性 核素的衰变对 MOFs 材料的辐照稳定性研究, 对未
来 MOFs 材料浓缩放射性核素的辐照稳定性和放射 性核素的释放对环境的污染, 需要深入研究。

(5) MOFs 材料的制备目前相对昂贵，在实际应 用中需要降低 MOFs 材料的制备成本。

(6) 对 MOFs 材料自身的环境污染及二次污染行 为需要深入研究。

(7) MOFs 的孔径对放射性核素在 MOFs 材料孔 道内的扩散迁移至关重要, 目前发表的 MOFs 材料 大多数孔道是微孔级 $(<2 \mathrm{~nm})$, 孔道尺寸的增大是一 个巨大挑战。 
表 2 上述主要吸附表征技术的目的、优点和缺点

Table 2 The main purpose, advantages and disadvantages of main adsorption characterization techniques mentioned above

\begin{tabular}{|c|c|c|c|}
\hline 技术 & 主要目的 & 优点 & 缺点 \\
\hline 宏观实验 & $\begin{array}{l}\text { 反应达到平衡所需时间, 最大 } \\
\text { 吸附量, 选择性和影响因素 }{ }^{[30]}\end{array}$ & $\begin{array}{l}\text { 非常直观得到实验结果, 方便和 } \\
\text { 有效 }\end{array}$ & 无法得到分子和原子水平上的作用机理 \\
\hline XPS 分析 & $\begin{array}{l}\text { 元素氧化态、元素种类和几乎所 } \\
\text { 有元素的键合关系(除了 } \mathrm{H} \text { 和 } \\
\mathrm{He})\end{array}$ & $\begin{array}{l}\text { 定量分析、元素组成分析、高表面 } \\
\text { 灵敏度检测(1 10 nm) }\end{array}$ & $\begin{array}{l}\text { 在真空中进行的测量, 可能改变样品的 } \\
\text { 性质; 在元素个数比值高于 } 0.05 \% \text { } \\
1.0 \% \text { 条件下进行, 依赖于元素的性质 }\end{array}$ \\
\hline XAFS 分析 & $\begin{array}{l}\text { 氧化态、配位数、原子间键距离 } \\
\text { 以及目标离子周围的离子状态 } \\
\text { [54] }\end{array}$ & $\begin{array}{l}\text { 特定的元素, 并且总是可以检测 } \\
\text { 到的, 对于研究非晶体材料是有 } \\
\text { 用的; 吸附物种的分析 }\end{array}$ & $\begin{array}{l}\text { 无法区分原子能相差较小的原子 }(\mathrm{C} 、 \mathrm{~N} \text { 、 } \\
\mathrm{O} \text { 或 } \mathrm{S} 、 \mathrm{Cl} 、 \mathrm{Mn} \text { 或 } \mathrm{Fe})^{[59-60]}\end{array}$ \\
\hline FT-IR 分析 & $\begin{array}{l}\text { 对微米范围内吸附行为的研究 } \\
\left(\text { 光密度 } \geqslant 10^{-5}\right)\end{array}$ & 灵敏检测官能团和极性键 ${ }^{[61]}$ & 定性而不是定量, 灵敏度低 \\
\hline DFT 计算 & $\begin{array}{l}\text { 键能、键长、轨道和系统电荷密 } \\
\text { 度 } \\
\text { [32,62] }\end{array}$ & $\begin{array}{l}\text { 对局部环境的吸附描述和原子级 } \\
\text { 吸附过程的描述 }{ }^{[63]}\end{array}$ & $\begin{array}{l}\text { 优化结构之间的能量与长时间模拟结果 } \\
\text { 较不准确 }\end{array}$ \\
\hline 分子动力学模拟 & 位置、势能和宏观现象的预测[64] & 吸附过程的快照在几秒内发生 ${ }^{[27]}$ & 长时间的计算时间, 依赖于计算的性能 \\
\hline
\end{tabular}

(8) 目前的研究基本都集中在基础研究领域, 在 实际复杂废水的处理中, 多种共存离子的存在对低 浓度放射性核素的高选择性吸附是至关重要的性质。 在未来的研究中, 对复杂体系条件下低浓度放射性 核素的高选择性吸附，对 MOFs 材料的实际应用，具 有重要的指导意义。

\section{参考文献:}

[1] AI Y J, LIU Y, LAN W Y, et al. The effect of $\mathrm{pH}$ on the U(VI) sorption on graphene oxide (GO): a theoretical study. Chemical Engineering Journal, 2018, 343: 460-466.

[2] PANG H W, WANG X X, YAO W, et al. Removal of radionuclides by metal oxide materials and mechanism research. Scientia Sinica Chimica, 2018, 48: 58-73.

[3] YANG S Y, WANG X X, CHEN Z S, et al. Synthesis of $\mathrm{Fe}_{3} \mathrm{O}_{4}$-based nanomaterials and their application in the removal of radionuclides and heavy metal ions. Progress in Chemistry, 2018, 30(2/3): 225-242.

[4] YU S J, WANG X X, PANG H W, et al. Boron nitride-based materials for the removal of pollutants from aqueous solutions: a review. Chemical Engineering Journal, 2018, 333: 343-360.

[5] YU S J, WANG X X, YANG S T, et al. Interaction of radionuclides with natural and manmade materials using XAFS technique. Science China Chemistry, 2016, 60(2): 170-187.

[6] LI X, LIU Y, ZHANG C L, et al. Porous $\mathrm{Fe}_{2} \mathrm{O}_{3}$ microcubes derived from metal organic frameworks for efficient elimination of organic pollutants and heavy metal ions. Chemical Engineering Journal, 2018, 336: 241-252.

[7] LIANG Y, GU P C, YAO W, et al. Adsorption of radionuclide uranium onto carbon-based nanomaterials from aqueous systems. Process in Chemistry, 2017, 29(9): 1062-1071.

[8] DU Y C, WANG X K, HOU R Q, et al. In-situ growth of $\mathrm{Nb}_{2} \mathrm{O}_{5}$ nanorods on diatomite and highly effective removal of $\mathrm{Cr}(\mathrm{VI})$. Journal of Inorganic Materials, 2018, 33(5): 557-564.

[9] CHEN H J, HUANG S Y, ZHANG Z B, et al. Synthesis of functional nanoscale zero-valent iron composites for the application of radioactive uranium enrichment from environment: a review. Acta Chimica Sinica, 2017, 75(6): 560-574.
[10] DU Y, WANG J, WANG H Q, et al. Research on sorption mechanism of radionuclides by manufactured nanomaterials. Journal of Agro-Environment Science, 2016, 35: 1837-1847.

[11] WANG X N, MENG H, MA F Y, et al. Influence of preparation method on oxidation degree of graphene oxide and adsorption for Th(IV) and U(VI). Journal of Inorganic Materials, 2016, 31(5): 454-460.

[12] GU P C, XING J L, WEN T, et al. Experimental and theoretical calculation investigation on efficient $\mathrm{Pb}$ (II) adsorption on etched $\mathrm{Ti}_{3} \mathrm{AlC}_{2}$ nanofibers and nanosheets. Environmental Science: Nano, 2018, 5(4): 946-955.

[13] YAO W, WU Y H, PANG H W, et al. In-situ reduction synthesis of manganese dioxide@polypyrrole core/shell nanomaterial for highly efficient enrichment of U(VI) and Eu(III). Science China Chemistry, 2018(7): 1-12.

[14] SONG S, YIN L, WANG X X, et al. Interaction of U(VI) with ternary layered double hydroxides by combined batch experiments and spectroscopy study. Chemical Engineering Journal, 2018, 338: 579-590.

[15] WANG P Y, YIN L, WANG X X, et al. L-cysteine intercalated layered double hydroxide for highly efficient capture of $\mathrm{U}(\mathrm{VI})$ from aqueous solutions. Journal of Environmental Management, 2018, 217: 468-477.

[16] LIU W, DAI X, BAI Z L, et al. Highly sensitive and selective uranium detection in natural water systems using a luminescent mesoporous metal-organic framework equipped with abundant lewis basic sites: a combined batch, X-ray absorption spectroscopy, and first principles simulation investigation. Environmental Science \& Technology, 2017, 51(7): 3911-3921.

[17] LI J, WANG X X, ZHAO G X, et al. Metal-organic frameworkbased materials: superior adsorbents for the capture of toxic and radioactive metal ions. Chemical Society Reviews, 2018, 47(7): 2322-2356.

[18] WU Y H, PANG H W, YAO W, et al. Synthesis of rod-like metal-organic framework (MOF-5) nanomaterial for efficient removal of $\mathrm{U}(\mathrm{VI})$ : batch experiments and spectroscopy study. Science Bulletin, 2018, 63(13): 831-839.

[19] DROUT R J, OTAKE K, HOWARTH A J, et al. Efficient capture of perrhenate and pertechnetate by a mesoporous $\mathrm{Zr}$ metal-organic framework and examination of anion binding motifs. Chemistry of Materials, 2018, 30(4): 1277-1284. 
[20] WANG Y L, LIU Z Y, LI Y X, et al. Umbellate distortions of the uranyl coordination environment result in a stable and porous polycatenated framework that can effectively remove cesium from aqueous solutions. Journal of America Chemistry Society, 2015, 137(19): 6144-6147.

[21] BANERJEE D, KIM D, SCHWEIGER M J, et al. Removal of $\mathrm{TcO}_{4}^{-}$ions from solution: materials and future outlook. Chemical Society Reviews, 2016, 45(10): 2724-2739.

[22] PANG H W, HUANG S Y, WU Y H, et al. Efficient elimination of U(VI) by polyethyleneimine decorated fly ash. Inorganic Chemistry Frontiers, 2018, 5: 2399-2407

[23] YU S J, WANG X X, TAN X L, et al. Sorption of radionuclides from aqueous systems onto graphene oxide-based materials: a review. Inorganic Chemistry Frontiers, 2015, 2(7): 593-612.

[24] DUAN L F, ZHANG Y, WANG L M, et al. Synthesis and characterization of $\mathrm{MnFe}_{2} \mathrm{O}_{4}$ with different morphologies and their application in water treatment. Journal of Inorganic Materials, 2014, 29(7): 763-768.

[25] CARBONI M, ABNEY C W, LIU S B, et al. Highly porous and stable metal-organic frameworks for uranium extraction. Chemical Science, 2013, 4(6): 2396-2402.

[26] BAI Z Q, YUAN L Y, ZHU L, et al. Introduction of amino groups into acid-resistant MOFs for enhanced U(VI) sorption. Journal of Materials Chemistry A, 2015, 3(2): 525-534.

[27] LI L N, MA W, SHEN S S, et al. A combined experimental and theoretical study on the extraction of uranium by amino-derived metal-organic frameworks through post-synthetic strategy. ACS Applied Materials \& Interfaces, 2016, 8(45): 31032-31041.

[28] ZHU L, SHENG D P, XU C, et al. Identifying the recognition site for selective trapping of ${ }^{99} \mathrm{TcO}_{4}^{-}$in a hydrolytically stable and radiation resistant cationic metal-organic framework. Journal of America Chemistry Society, 2017, 139(42): 14873-14876.

[29] SHENG D P, ZHU L, XU C, et al. Efficient and selective uptake of $\mathrm{TcO}_{4}{ }^{-}$by a cationic metal-organic framework material with open $\mathrm{Ag}^{+}$sites. Environmental Science \& Technology, 2017, 51(6): 3471-3479.

[30] YANG P P, LIU Q, LIU J Y, et al. Interfacial growth of a metal-organic framework (UiO-66) on functionalized graphene oxide (GO) as a suitable seawater adsorbent for extraction of uranium(VI). Journal of Materials Chemistry A, 2017, 5(34): 17933-17942.

[31] ZHANG N, YUAN L Y, GUO W L, et al. Extending the use of highly porous and functionalized MOFs to Th(IV) capture. ACS Applied Materials \& Interfaces, 2017, 9(30): 25216-25224.

[32] PENG Y G, HUANG H L, LIU D H, et al. Radioactive barium ion trap based on metal-organic framework for efficient and irreversible removal of barium from nuclear wastewater. ACS Applied Materials \& Interfaces, 2016, 8(13): 8527-8535.

[33] YUAN G Y, TIAN Y, LIU J, et al. Schiff base anchored on metal-organic framework for $\mathrm{Co}$ (II) removal from aqueous solution. Chemical Engineering Journal, 2017, 326: 691-699.

[34] GAO Y J, FENG M L, ZHANG B, et al. An easily synthesized microporous framework material for the selective capture of radioactive $\mathrm{Cs}^{+}$and $\mathrm{Sr}^{2+}$ ions. Journal of Materials Chemistry A, 2018, 6(9): 3967-3976

[35] YAO W, WANG X X, LIANG Y, et al. Synthesis of novel flower-like layered double oxides/carbon dots nanocomposites for $\mathrm{U}(\mathrm{VI})$ and ${ }^{241} \mathrm{Am}(\mathrm{III})$ efficient removal: batch and EXAFS studies. Chemical Engineering Journal, 2018, 332: 775-786.

[36] WANG X X, YU S J , WU Y H, et al. The synergistic elimination of uranium(VI) species from aqueous solution using bi-functional nanocomposite of carbon sphere and layered double hydroxide.
Chemical Engineering Journal, 2018, 342: 321-330.

[37] WANG X X, LIU Y, PANG H W, et al. Effect of graphene oxide surface modification on the elimination of $\mathrm{Co}(\mathrm{II})$ from aqueous solutions. Chemical Engineering Journal, 2018, 344: 380-390.

[38] YU S J, WANG J, SONG S, et al. One-pot synthesis of graphene oxide and Ni-Al layered double hydroxides nanocomposites for the efficient removal of U(VI) from wastewater. Science China Chemistry, 2017, 60(3): 415-422.

[39] YU S J, WANG X X, CHEN Z S, et al. Interaction mechanism of radionickel on Na-montmorillonite: influences of $\mathrm{pH}$, electrolyte cations, humic acid and temperature. Chemical Engineering Journal, 2016, 302: 77-85.

[40] YIN L, SONG S, WANG X X, et al. Rationally designed core-shell and yolk-shell magnetic titanate nanosheets for efficient U(VI) adsorption performance. Environmental Pollution, 2018, 238: 725-738.

[41] GU P C, ZHANG S, LI X, et al. Recent advances in layered double hydroxide-based nanomaterials for the removal of radionuclides from aqueous solution. Environmental Pollution, 2018, 240: 493-505.

[42] SONG W C, WANG X X, CHEN Z S, et al. Enhanced immobilization of U(VI) on Mucor circinelloides in presence of $\mathrm{As}(\mathrm{V})$ : batch and XAFS investigation. Environmental Pollution, 2018, 237: 228-236.

[43] TAN X L, FANG M, TAN L Q, et al. Core-shell hierarchical $\mathrm{C} @ \mathrm{Na}_{2} \mathrm{Ti}_{3} \mathrm{O}_{7} \cdot 9 \mathrm{H}_{2} \mathrm{O}$ nanostructures for the efficient removal of radionuclides. Environmental Science: Nano, 2018, 5(5): 1140-1149.

[44] NAEIMI S, FAGHIHIAN H. Performance of novel adsorbent prepared by magnetic metal-organic framework MOF modified by potassium nickel hexacyanoferrate for removal of $\mathrm{Cs}^{+}$from aqueous solution. Separation and Purification Technology, 2017, 175: 255-265.

[45] ZHANG C L, LIU Y, LI X, et al. Highly uranium elimination by crab shells-derived porous graphitic carbon nitride: batch, EXAFS and theoretical calculations. Chemical Engineering Journal, 2018, 346: 406-415.

[46] HU Y Z, WANG X X, ZOU Y D, et al. Superior sorption capacities of $\mathrm{Ca}-\mathrm{Ti}$ and $\mathrm{Ca}-\mathrm{Al}$ bimetallic oxides for U(VI) from aqueous solutions. Chemical Engineering Journal, 2017, 316: 419-428.

[47] CHEN Z S, WANG J, PU Z X, et al. Synthesis of magnetic $\mathrm{Fe}_{3} \mathrm{O}_{4} / \mathrm{CFA}$ composites for the efficient removal of U(VI) from wastewater. Chemical Engineering Journal, 2017, 320: 448-457.

[48] ZHANG C L, LI X, CHEN Z S, et al. Synthesis of ordered mesoporous carbonaceous materials and its highly efficient capture of uranium from solutions. Science China Chemistry, 2018, 61(3): 281-293.

[49] SUN Y B, LU S H, WANG X X, et al. Plasma-facilitated synthesis of amidoxime/carbon nanofiber hybrids for effective enrichment of ${ }^{238} \mathrm{U}(\mathrm{VI})$ and ${ }^{241} \mathrm{Am}(\mathrm{III})$. Environmental Science \& Technology, 2017, 51(21): 12274-12282.

[50] WANG J, WANG X X, ZHAO G X, et al. Polyvinylpyrrolidone and polyacrylamide intercalated molybdenum disulfide as adsorbents for enhanced removal of chromium(VI) from aqueous solutions. Chemical Engineering Journal, 2018, 334: 569-578.

[51] YANG D X, WANG X X, SONG G, et al. One-pot synthesis of arginine modified hydroxyapatite carbon microsphere composites for efficient removal of U(VI) from aqueous solutions. Science Bulletin, 2017, 62(23): 1609-1618.

[52] SONG S, HUANG S Y, ZHANG R, et al. Simultaneous removal of $\mathrm{U}(\mathrm{VI})$ and humic acid on defective $\mathrm{TiO}_{2-x}$ investigated by batch and spectroscopy techniques. Chemical Engineering Journal, 2017, 325: 576-587.

[53] TAN L Q, TAN X L, MEI H Y, et al. Coagulation behavior of humic acid in aqueous solutions containing $\mathrm{Cs}^{+}, \mathrm{Sr}^{2+}$ and $\mathrm{Eu}^{3+}$ : DLS, 
EEM and MD simulation. Environmental Pollution, 2018, 235: 835-843.

[54] ZHANG J Y, ZHANG N, ZHANG L J, et al. Adsorption of uranyl ions on amine-functionalization of MIL-101(Cr) nanoparticles by a facile coordination-based post-synthetic strategy and X-ray absorption spectroscopy studies. Scientific Reports, 2015, 5: 13514$1-10$.

[55] WEN H, PAN Z Z, GIAMMAR D E, et al. Enhanced uranium immobilization by phosphate amendment under variable geochemical and flow conditions: insights from reactive transport modeling. Environmental Science \& Technology, 2018, 52(10): 5841-5850.

[56] ZHANG Y J, LAN J H, WANG L, et al. Adsorption of uranyl species on hydroxylated titanium carbide nanosheet: a first-principles study. Journal of Hazardous Materials, 2016, 308: 402-410.

[57] WANG L, YUAN L Y, CHEN K, et al. Loading actinides in multilayered structures for nuclear waste treatment: the first case study of uranium capture with vanadium carbide MXene. ACS Applied Materials \& Interfaces, 2016, 8(25): 16396-16403.

[58] ZHENG T, YANG Z X, GUI D X, et al. Overcoming the crystallization and designability issues in the ultrastable zirconium phos- phonate framework system. Nature Communications, 2017, 8: 15369-1-11.

[59] SHENG G D, SHAO D D, FAN Q H D, et al. Effect of pH and ionic strength on sorption of Eu(III) to MX-80 bentonite: batch and XAFS study. Radiochimica Acta, 2009, 97(11): 621-630.

[60] TAN X L, REN X M, CHEN C L, et al. Analytical approaches to the speciation of lanthanides at solid-water interfaces. TrAC Trends in Analytical Chemistry, 2014, 61: 107-132.

[61] LUO F, CHEN J L, DANG L L, et al. High-performance $\mathrm{Hg}^{2+}$ removal from ultra-low-concentration aqueous solution using both acylamide-and hydroxyl-functionalized metal-organic framework. Journal of Materials Chemistry A, 2015, 3(18): 9616-9620.

[62] BANERJEE D, XU W Q, NIE Z M, et al. Zirconium-based metal-organic framework for removal of perrhenate from water. Inorganic Chemistry, 2016, 55(17): 8241-8243.

[63] HOSKINS B F, ROBSON R. Infinite polymeric frameworks consisting of three dimensionally linked rod-like segments. Journal of the American Chemical Society, 1989, 111(15): 5962-5964.

[64] NALAPARAJU A, JIANG J W. Ion exchange in metal-organic framework for water purification: insight from molecular simulation. The Journal of Physical Chemistry C, 2012, 116(12): 6925-6931. 